\title{
On the Zone of a Surface in a Hyperplane Arrangement*
}

\author{
Boris Aronov, ${ }^{1}$ Marco Pellegrini, ${ }^{2}$ and Micha Sharir ${ }^{2,3}$ \\ 'Department of Computer Science, Polytechnic University, \\ Brooklyn, NY 11201, USA \\ ${ }^{2}$ Courant Institute of Mathematical Sciences, New York University, \\ New York, NY 10012, USA \\ ${ }^{3}$ School of Mathematical Sciences, Tel Aviv University, Tel Aviv, Israel
}

\begin{abstract}
Let $H$ be a collection of $n$ hyperplanes in $\mathbb{R}^{d}$, let $\mathscr{A}$ denote the arrangement of $H$, and let $\sigma$ be a $(d-1)$-dimensional algebraic surface of low degree, or the boundary of a convex set in $\mathbb{R}^{d}$. The zone of $\sigma$ in $\mathscr{A}$ is the collection of cells of $\mathscr{N}$ crossed by $\sigma$. We show that the total number of faces bounding the cells of the zone of $\sigma$ is $O\left(n^{d-1} \log n\right)$. More generally, if $\sigma$ has dimension $p, 0 \leq p<d$, this quantity is $O\left(n^{\lfloor(d+p) / 2\rfloor}\right)$ for $d-p$ even and $O\left(n^{\lfloor(d+p) / 2\rfloor} \log n\right)$ for $d-p$ odd. These bounds are tight within a logarithmic factor.
\end{abstract}

\section{Introduction}

A set $H$ of $n$ hyperplanes in $d$-dimensional space $\mathbb{R}^{d}$ decomposes $\mathbb{R}^{d}$ into open cells of dimension $d$ (also called $d$-faces) and into relatively open faces of dimension $k$, $0 \leq k<d$. These cells and faces define a cell complex which is commonly known as the arrangement $\mathscr{A}=\mathscr{A}(H)$ of $H$. We define the complexity of a cell in $\alpha$ to be the number of faces that are contained in the closure of the cell.

\footnotetext{
* This paper is the union of two conference proceedings papers [3], [15]. Work on this paper by M. Pellegrini and M. Sharir has been supported by NSF Grant CCR-8901484. Work on this paper by M. Sharir has also been supported by ONR Grant NO0014-90-J-1284 and by grants from the U.S.-Israeli Binational Science Foundation, the G.I.F. (the German-Israeli Foundation for Scientific Research and Development), and the Fund for Basic Research administered by the Israeli Academy of Sciences. M. Pellegrini's current address is Department of Computing, King's College, Strand, London WC2R 2LS, England.
} 
Let $\sigma$ be an arbitrary subset of $\mathbb{R}^{d}$. We define $\mathscr{Z}_{\sigma}(H)$, the zone of $\sigma$ in $\mathscr{A}$, to be the set of all (open) cells in $\mathscr{A}$ that intersect $\sigma$; the complexity of a zone is the sum of the complexities of its constituent cells. In the following analysis we concentrate on the case where $\sigma$ is either a $p$-dimensional algebraic surface of degree $\delta$ in $\mathbb{R}^{d}$, where $\delta$ is a small constant and $0 \leq p<d$, or the boundary of a convex set of affine dimension $p+1$, for the same range of values of $p$. However, most of our analysis, with the notable exception of Lemmas 2.2 and 2.3, holds for an arbitrary set $\sigma$.

For technical reasons that will become more apparent later, we prefer to view $\mathscr{A}$ as an object in $d$-dimensional projective space, rather than in $\mathbb{R}^{d}$. Equivalently, we regard a pair of antipodal cells in $\mathscr{A}$ as one cell. It is easily checked that this assumption does not affect the asymptotic behavior of the zone complexity; however, together with general position assumptions mentioned below, it allows us to view each face of $\mathscr{A}$ as a simple convex polytope, thus making separate treatment of unbounded faces unnecessary most of the time.

A fundamental result on hyperplane arrangements is the Zone Theorem [7], in which $\sigma$ is assumed to be a hyperplane distinct from those in $H$. It asserts that the zone of a hyperplane in an arrangement of $n$ hyperplanes in $\mathbb{R}^{d}$ has complexity $\Theta\left(n^{d-1}\right)$. A recent proof of the Zone Theorem is given in [9].

In this paper we extend the Zone Theorem to cases where $\sigma$ is a more general set, as described above. Specifically, we show:

Theorem 1.1 (Extended Zone Theorem). The complexity of the zone of a $(d-1)$ dimensional surface $\sigma$, which is either a small-degree algebraic surface or the boundary of an arbitrary convex set, in an arrangement of n hyperplanes in $\mathbb{R}^{d}$ is $O\left(n^{d-1} \log n\right)$, where $d \geq 3$ and the constant of proportionality depends on $d$ and on the degree $\delta$ of $\sigma$. More generally, if $\sigma$ is a p-dimensional algebraic surface of small degree, or the relative boundary of a convex set of affine dimension $p+1$, for $0 \leq p<d$, the complexity of the zone of $\sigma$ is $O\left(n^{\lfloor(d+p) / 2\rfloor}\right)$ if $d-p$ is even and $O\left(n^{\lfloor(d+p) / 2\rfloor} \log n\right)$ if it is odd.

We note that when $d=2$, a somewhat better bound of $O(n \alpha(n))$ is known for the complexity of the zone of an algebraic curve in a line arrangement [8], where $\alpha(n)$ is the inverse Ackermann function. We have so far been unable to obtain similarly improved bounds in higher dimensions. We do not even know if the bound $O(n \alpha(n))$ is tight in the worst case in the plane. The best known lower bound, in any dimension $d$, is $\Omega\left(n^{\lfloor(d+p) / 2\rfloor}\right)$ [11], so there remains a small gap between the lower and upper bounds. Related results for planar arrangements have been obtained by Bern et al. [5], and for zones of p-flats by Houle and Tokuyama [11]; the latter bounds are the same as we get in Theorem 1.1. It has been pointed out by Houle and Tokuyama [11] that the definition of the zone of a surface as a collection of open (rather than closed) cells of the arrangement is crucial for the theorem to hold for low-dimensional surfaces; with the alternative definition, in an arrangement $n$ of hyperplanes passing through a common point $v$, the zone of $v$ would include all cells of the arrangement, providing an immediate $\Omega\left(n^{d-1}\right)$ lower bound on the worst-case zone complexity for a zero-dimensional object!

The main difference between the zone of a hyperplane and that of a more 
general surface $\sigma$ is in the behavior of what we call popular facets. These are facets (i.e., $(d-1)$-dimensional faces) $f$ that bound two adjacent cells $C, C^{\prime}$ of $\mathscr{A}$ so that both $C$ and $C^{\prime}$ belong to the zone of $\sigma$. Even though popular facets do exist in the zone of a hyperplane, there they must always meet the zone hyperplane, which is not necessarily the case for zones of curved surfaces. A main step in our analysis is to obtain a sharp bound on the number and complexity of the popular facets in a zone. To this end we extend the notion of popularity to faces of any dimension and derive a recurrence for the complexity of these popular faces.

\section{Proof of the Extended Zone Theorem}

For a $d$-polyhedron $P$ let $f_{k}(P)$ denote the number of $k$-faces of $P$ (i.e., faces of dimension $k$ ). For $0 \leq k \leq d$, let $z_{k}(\sigma ; H)$ denote $\sum_{C \in z_{f}(H)} f_{k}(\bar{C})$, where $\bar{C}$ denotes the topological closure of cell $C$. Finally, for $n>0, d>0$, and $0 \leq k \leq d$, let $z_{k}^{(p, \delta)}(n, d)$ denote the maximum of $z_{k}(\sigma ; H)$ over all $p$-dimensional surfaces $\sigma$ of degree at most $\delta$ and all sets $H$ of $n$ hyperplanes in $\mathbb{R}^{d}$; to reduce proliferation of indices, we omit the superscript $(p, \delta)$ in what follows.

As we are interested in the asymptotic behavior of $z_{k}$, we assume $n>d$ throughout the proof, unless stated otherwise.

First note that $z_{k}(\sigma ; H)$ achieves its maximum when $\sigma$ and $H$ are in general position, i.e., every $j \leq d$ hyperplanes in $H$ intersect in a $(d-j)$-flat, no $d+1$ hyperplanes have a point in common, and $\sigma$ is not tangent to any flat formed by the intersection of $j \leq d$ hyperplanes of $H$ and does not meet any such $j$-flat, for $0 \leq j<d-p$. (Recall that $\mathscr{A}(H)$ is viewed as a projective arrangement, so we require that the above general position assumptions hold at "points at infinity" as well.) This can be proved using a standard perturbation argument: displacing the hyperplanes of $H$ slightly will put $\sigma$ and $H$ in general position, and can only increase the complexities of the cells in $\mathscr{Z}_{\sigma}(H)$, through vertex truncation or the actions dual to vertex pulling or pushing (see pp. 78-83 of [10]).

Let $H$ be a set of $n$ hyperplanes in $\mathbb{R}^{d}$, and let $\sigma$ be an algebraic surface as above, so that $\sigma$ and $H$ are in general position. A $k$-face $f$ in $\mathscr{A}(H)$ now lies in exactly $d-k$ hyperplanes of $H$ and is part of the boundary of $2^{d-k}$ cells of $\mathscr{A}(H)$. More than one of those cells can lie in $\mathscr{Z}_{\sigma}(H)$, and thus the contribution of $f$ to $z_{k}(\sigma ; H)$ can be larger than one. In order to have entities that contribute at most one to the count $z_{k}(\sigma ; H)$ we define a $k$-border to be a pair $(f, C)$, where $f$ is a $k$-face in $\mathscr{A}(H)$ and $C$ is a cell that has $f$ on its boundary. Thus $z_{k}(\sigma ; H)$ counts all borders of dimension $k$ in $\mathscr{Z}_{\sigma}(H)$, i.e., $k$-borders $(f, C)$ with $C \in \mathscr{Z}_{\sigma}(H)$. More generally, for $0 \leq k \leq i \leq d, \mathbf{a}(k, i)$-border is a pair of faces $(f, g)$ in $\alpha$ of dimension $k$ and $i$, respectively, with $f \subseteq \bar{g}$. We refer to a pair of faces, $f, g$, with $f \subseteq \bar{g}$, as incident faces. Note that $k$-borders defined above are simply $(k, d)$-borders.

We call a $k$-face $f$ in $\mathscr{A}$ popular if all $2^{d-k}$ cells in $d$ incident to $f$ belong to $\mathscr{Z}_{a}(H)$. Note that a "popular cell" is simply a zone cell, i.e., a cell of,$d$ met by $\sigma$.

A $(k, i)$-border $(f, g)$ is popular if $g$ is a popular $i$-face. Let $\tau_{k}^{(i)}(\sigma ; H)$ be the number of popular $(k, i)$-borders. Notice that the problem of bounding the complexity of the zone of $\sigma$ in $\mathscr{d}$ reduces to bounding the quantities $\tau_{k}^{(d)}(\sigma ; H)$, 
for all $0 \leq k \leq d$, as $z_{k}(\sigma ; H)=\tau_{k}^{(d)}(\sigma ; H)$. We obtain such bounds by inductively estimating $\tau_{k}^{(i)}$ for all $0 \leq k \leq i \leq d$. We begin by providing a bound on $\tau_{k}^{(k)}$ for all $0 \leq k \leq d$.

Lemma 2.1. Let $H$ be a collection of $n$ hyperplanes in general position in $\mathbb{R}^{d}$. Then, for any set $X \subset \mathbb{R}^{d}$ and $0 \leq k \leq d$,

$$
\tau_{k}^{(k)}(X ; H) \leq 2\left(\begin{array}{l}
d \\
k
\end{array}\right) \tau_{d}^{(d)}(X ; H)
$$

Proof. Let $k<d$. Recall that $\tau_{k}^{(k)}(X ; H)$ is simply the number of popular $k$-faces, i.e., $k$-faces $f$ for which all $2^{d-k}$ incident cells belong to the zone of $X$. To prove the lemma, it is sufficient to associate each such face with one of the incident cells, and argue that no zone cell gets charged more than $2\left({ }_{k}^{d}\right)$ times.

We set up the correspondence as follows: First observe that the notion of popularity depends only on the set of arrangement cells that are met by $X$. Thus picking one point of $X$ in each such cell and discarding the rest of $X$ does not affect the statement of the lemma. Now construct a large simplex $\Delta$ in generic position that encloses all bounded cells and faces of $\mathscr{A}(H)$, and meets all unbounded faces. We now replace $\mathscr{A}(H)$ by $\mathscr{A}\left(H^{+}\right)$, where $H^{+}$is obtained by adding to $H$ the $d+1$ hyperplanes defining $\Delta$, but only consider the portion of $\mathscr{A}\left(H^{+}\right)$contained in $\bar{\Delta}$. We tag each cell of $\mathscr{A}\left(H^{+}\right)$within $\bar{\Delta}$ as a zone cell either if it is a bounded cell of $\mathscr{Z}_{X}(H)$ or if it is contained in an unbounded cell of $\mathscr{Z}_{X}(H)$. Since we consider the original arrangement in projective space, it follows that each unbounded zone cell in $\mathscr{A}(H)$ tags two "antipodal" bounded cells in $\mathscr{A}\left(H^{+}\right)$as zone cells. Let $\mathscr{Z}_{X}\left(H^{+}\right)$denote the collection of all tagged zone cells in $\mathscr{A}\left(H^{+}\right)$. Note that to each bounded face of $\mathscr{Z}_{X}(H)$ there corresponds a unique bounded face of $\mathscr{Z}_{X}\left(H^{+}\right)$, and to each unbounded face of $\mathscr{Z}_{X}(H)$ there correspond two distinct bounded faces of $\mathscr{A}\left(H^{+}\right)$.

Rotate the new arrangement in such a fashion that every face has a unique lowest vertex, with the height measured in terms of the $x_{d}$ coordinate. We claim that, since $\mathscr{A}=\mathscr{A}\left(\mathrm{H}^{+}\right)$is a simple arrangement, the lowest vertex $v_{f}$ of a face $f$ is the lowest vertex of exactly one of the cells incident to $f$. The way to see this is to observe that, among all cells incident to $v_{f}$, the unique cell that has $v_{f}$ as its lowest vertex has the property that its bounding faces incident to $v_{f}$ are exactly those faces of $\mathscr{A}$ that are incident to $v_{f}$ and have $v_{f}$ as their lowest vertex.

Note that each popular $k$-face in the original projective arrangement is mapped to one or two new popular $k$-faces in $\mathscr{A}\left(H^{+}\right)$(two if the original face was unbounded). In the latter case we arbitrarily pick one of the two new faces. Each new popular $k$-face is assigned to a unique cell with which it shares its lowest vertex. No cell in $\mathscr{Z}_{X}\left(H^{+}\right)$is charged more than $\left(\begin{array}{l}d \\ k\end{array}\right)$ times, as this is the total number of $k$-faces in $\mathscr{A}\left(H^{+}\right)$sharing its lowest vertex, since $\mathscr{A}\left(H^{+}\right)$is a simple arrangement. By the above remark, the number of cells in $\mathscr{Z}_{x}\left(H^{+}\right)$is at most twice the number of cells in $\mathscr{Z}_{X}(H)$, thus completing the proof of the lemma.

Lemma 2.2. Let $H$ be a collection of $n$ hyperplanes in $\mathbb{R}^{d}$ and let $\sigma$ be an algebraic surface of dimension $p$ and degree $\delta$. Assume that $H$ and $\sigma$ are in general position. 
Then

$$
\tau_{k}^{(k)}(\sigma ; H)=O\left(n^{p}\right), \quad 0 \leq k \leq d,
$$

where the constant of proportionality depends on $k, d$, and $\delta$; the dependence on $\delta$ is $O\left(\delta^{d}\right)$.

Proof. By Lemma 2.1, it is sufficient to show that $\tau_{d}^{(d)}(\sigma ; H)$ is $O\left(n^{p}\right)$, i.e., $\sigma$ meets $O\left(n^{p}\right)$ cells of $\mathscr{A}$. We charge each cell $C$ of $\mathscr{X}_{\sigma}(H)$ to a $k$-flat $F$ formed by the intersection of some $d-k$ hyperplanes of $H$, so that $F \cap \sigma$ meets $\bar{C}$ and $k \leq d$ is the smallest integer for which this property holds. It follows that $k+p \geq d$ from our assumptions on general position. Thus $F$ contains a face $f$ of $\bar{C}$ of dimension $k$, so that $\sigma \cap f \neq \varnothing$ but $\sigma$ does not meet the relative boundary of $f$. Then $f$ contains one or more connected components of $\sigma \cap F$. However $\sigma \cap F$ is an algebraic surface in $F$ of degree at most $\delta$, so it has a constant number (which by Milnor's theorem [12] is $O\left(\delta^{k}\right)=O\left(\delta^{d}\right)$ ) of connected components. Thus, over all cells $C$ of $\mathscr{Z}_{\sigma}(H)$, $F$ has only a constant number of faces $f$ of this form, and each such face bounds at most $2^{d-k} \leq 2^{p}$ cells of $\mathscr{Z}_{\sigma}(H)$. Since the number of $k$-flats, for $d-p \leq k \leq d$, formed by intersections of the hyperplanes of $H$, is $O\left(n^{p}\right)$, it follows that the total number of cell-charges is $O\left(n^{p}\right)$. This establishes the claim of the lemma.

Lemma 2.3. Let $H$ be a collection of $n$ hyperplanes in general position in $\mathbb{R}^{d}$. Then $\tau_{k}^{(k)}(\sigma ; H)=O\left(n^{p}\right)$, whenever $\sigma$ is the boundary of an arbitrary convex set of affine dimension $p+1$.

Proof. The claim is immediate by noting that the argument in the proof of Lemma 2.2 applies to the case of the boundary of a convex set as well, since the number of connected components of $\sigma \cap F$, for any flat $F$, is at most 2 .

Note. The upper bounds in Lemmas 2.2 and 2.3 are easily seen to be asymptotically tight - take $\sigma$ to be a generic $p$-flat and notice that every $l$-face of the arrangement induced by $\mathscr{A}$ in $\sigma$ corresponds to a popular $(l+d-p)$-face in $\mathscr{A}$. It remains to argue the lower bound on $\tau_{k}^{(k)}$ for $0 \leq k<d-p$. In fact, it is easily verified that, as long as $\sigma$ is a $p$-flat and the general position assumptions hold, $\tau_{k}^{(k)}(\sigma ; H)=0$ for $k<d-p$. For example, when $p=d-1$, a hyperplane cannot cut all $2^{d}$ "octants" incident to a vertex of $\mathscr{A}(H)$-hence there are no popular vertices. The situation changes drastically when $\sigma$ is allowed to be a more general algebraic surface. For example, for $p=d-1$, let $\sigma$ be the union of two parallel hyperplanes lying on either side of a hyperplane $h \in H$ and very close to it. It is easily checked that all vertices of $\mathscr{A}(H)$ contained in $h$ are popular, thereby showing $\tau_{0}^{(0)}(\sigma ; H)=\Omega\left(n^{d-1}\right)$. The lower bound on $\tau_{k}^{(k)}$, for $k<d-p$ and general $p<d-1$, can be obtained by a slight modification of this argument, with the two hyperplanes replaced by a cylinder around a $p$-flat of the arrangement.

Corollary 2.4. For any algebraic surface $\sigma \subset \mathbb{R}^{d}$ and a set $H$ of $n$ hyperplanes, $z_{d}(\sigma ; H)=O\left(n^{\mathrm{dim} \sigma}\right)$ with the constant of proportionality depending on $d$ and on the degree $\delta$ of $\sigma$. The assertion also holds for the boundary of an arbitrary convex set. 
Proof. As we already noted, $z_{d}(\sigma ; H)$ is maximized when $\sigma$ and $H$ are in general position. Now recall that $z_{d}(\sigma ; H)=\tau_{d}^{(d)}(\sigma ; H)$ by definition.

We now proceed by induction on $i$, and derive a recurrence for $\tau_{k}^{(i)}(\sigma ; H)$, for $0 \leq k<i$, using an approach similar to that used in [9] and also in [2]. In more detail, fix a hyperplane $h \in H$ and consider all popular $(k, i)$-borders $\left(f_{0}, g_{0}\right)$ in $\mathscr{Z}_{\sigma}(H)$ with $f_{0} \not \subset h$. When we remove $h$, the face $g_{0}$ becomes part of a possibly larger $i$-face $g$, which is clearly also popular (in the reduced arrangement). Moreover, $f_{0}$ is a part of some $k$-face contained in $\bar{g}$. So let $(f, g)$ be a popular $(k, i)$-border in $\mathscr{Z}_{\sigma}(H \backslash\{h\})$, and consider what happens to it when $h$ is reinserted into the arrangement. Let $C_{l}, l=1, \ldots, 2^{d-i}$, be the cells in $\mathscr{Z}_{a}(H \backslash\{h\})$ incident to $g$. The following cases may occur:

$h \cap g=\varnothing:$ In this case $g$ may or may not be popular in $\mathscr{Z}_{\sigma}(H)$, but $(f, g)$ contributes at most one popular $(k, i)$-border to this zone, namely itself.

$h \cap g \neq \varnothing$ and $h \cap f=\varnothing$ : Again, $(f, g)$ can contribute at most one popular $(k, i)$-border to $\mathscr{Z}_{\sigma}(H)$, namely $\left(f, g^{+}\right)$, where $g^{+}$is the portion of $g$ lying to the same side of $h$ as $f$.

$h \cap g \neq \varnothing$ and $h \cap f \neq \varnothing$ : Let $h^{+}, h^{-}$denote the two open half-spaces bounded by $h$, and consider the two $(k, i)$-borders $\left(f \cap h^{+}, g \cap h^{+}\right)$and $\left(f \cap h^{-}, g \cap h^{-}\right)$. We are only interested in the case where both of them become popular borders in $\mathscr{Z}_{\sigma}(H)$, for only then will our count go up. Let $C_{l}^{+}=C_{1} \cap h^{+}$and $C_{l}^{-}=C_{1} \cap h^{-}$for $l=1, \ldots, 2^{d-i}$. Thus we are interested in situations where $\sigma$ meets all $2^{d-i+1}$ cells $C_{l}^{+}, C_{l}^{-}$. Notice that all these cells are incident to $g \cap h$, an $(i-1)$-face in $\mathscr{A}$. Hence $g \cap h$ is a popular face and $(f \cap h, g \cap h)$ is a popular $(k-1, i-1)$-border in $\mathscr{Z}_{\sigma}(H)$.

To sum up, the number of popular $(k, i)$-borders in $\mathscr{Z}_{\sigma}(H)$ which are not contained in $h$ is bounded by

$$
\tau_{k}^{(i)}\left(\sigma ; H \backslash\left\{h_{\xi}\right)+\rho_{h}\right.
$$

where $\rho_{h}$ is the number of popular $(k-1, i-1)$-borders $\left(f^{\prime}, g^{\prime}\right)$ with $g^{\prime} \subset h$. If we sum these bounds over all hyperplanes $h \in H$ and observe that every popular $(k, i)$-border in $\mathscr{Z}_{\sigma}(H)$ is counted exactly $n-d+k$ times (it is not counted if and only if $h$ is one of the $d-k$ hyperplanes containing the $k$-face of the border), we obtain, similar to [9],

$$
(n-d+k) \tau_{k}^{(i)}\left(\sigma ; \leq \sum_{h \in H} \tau_{k}^{(i)}(\sigma ; H \backslash\{h\})+(d-i+1) \tau_{k-1}^{(i-1)}(\sigma ; H),\right.
$$

where the factor $(d-i+1)$ comes from the fact that a popular $(k-1, i-1)$ border is charged $d-i+1$ times, once for each hyperplane $h$ containing it.

For the sake of clarity of exposition, we first solve the recurrence for $p=d-1$, and then discuss the easy extension to general values of $p$. Also, we only handle the case of an algebraic surface, since the case of a convex surface can be treatcd in much the same way.

For a fixed number $\delta$, let us denote by $\tau_{h}^{(i)}(n, d)$ the maximum of $\tau_{h}^{(i)}(\sigma ; /)$ over 
all choices of a set $H$ of $n$ hyperplanes in $\mathbb{R}^{d}$ and an algebraic surface $\sigma$ of degree at most $\delta$ and dimension $d-1$, with $H$ and $\sigma$ in general position. We thus have

$$
\tau_{k}^{(k)}(n, d)=O\left(n^{d-1}\right), \quad 0 \leq k \leq d
$$

and

$\tau_{k}^{(i)}(n, d) \leq \frac{n}{n-d+k} \tau_{k}^{(i)}(n-1, d)+\frac{d-i+1}{n-d+k} \tau_{k-1}^{(i-1)}(n, d), \quad 0 \leq k<i \leq d$.

When $k=0$, the rightmost term in (2) vanishes, but the recurrence solves to $O\left(n^{d}\right)$ (see [2] and [9]), which is too large for our purposes. However, we observe that, trivially, $\tau_{0}^{(i)}(n, d) \leq 2 \tau_{1}^{(i)}(n, d)$. Thus it suffices to analyze (2) only for $k \geq 1$.

We first transform the relation (2) into a simpler one, by substituting

$$
\tau_{k}^{(i)}(n, d)=\left(\begin{array}{c}
d \\
d-k
\end{array}\right) \psi_{k}^{(i)}(n, d)
$$

(Recall that we have assumed that $n>d$.) This yields the following relations, as is easily verified:

$$
\psi_{k}^{(k)}(n, d)=O\left(n^{k-1}\right), \quad 1 \leq k \leq d
$$

and

$$
\psi_{k}^{(i)}(n, d) \leq \psi_{k}^{(i)}(n-1, d)+\frac{d-i+1}{d-k+1} \psi_{k-1}^{(i-1)}(n, d), \quad 1 \leq k<i \leq d
$$

Our goal is now to show that $\psi_{k}^{(i)}(n, d)=O\left(n^{k-1} \log n\right)$. We prove this by induction on $i$. The base case $i=0$ only allows $k=0$, and we have already shown that $\tau_{0}^{(0)}(n, d)=O\left(n^{d-1}\right)$, and thus $\psi_{0}^{(0)}(n, d)=O\left(n^{k-1}\right)$. Similarly, the case $i=1$ also follows from (1), since we only consider the case $k \geq 1$.

The case $i=2$ is the most interesting one, since it is there where the $\log n$ factor enters our analysis. To be more precise, the interesting case is $i=2, k=1$, as the case $k=2$ has already been dealt with in (1). In this special case, (3) becomes

$$
\psi_{1}^{(2)}(n, d) \leq \psi_{1}^{(2)}(n-1, d)+{ }_{d}^{d-1} \psi_{0}^{(1)}(n, d)
$$

However, we have already shown that

$$
\psi_{0}^{(1)}(n, d)=\frac{1}{\left(\begin{array}{l}
n \\
d
\end{array}\right)} \tau_{0}^{(1)}(n, d) \leq \frac{2}{\left(\begin{array}{l}
n \\
d
\end{array}\right)} \tau_{1}^{(1)}(n, d)=O\left(\frac{1}{n}\right)
$$


Thus we obtain the recurrence

$$
\psi_{1}^{(2)}(n, d)=\psi_{1}^{(2)}(n-1, d)+o\left(\frac{1}{n}\right)
$$

whose solution is $\psi_{1}^{(2)}(n, d)=O(\log n)$, as asserted.

For $i>2$, we first ignore both cases $k=0$ and $k=1$. By induction hypothesis on $i$ we obtain the following recurrence for $k<i$ :

$$
\psi_{k}^{(i)}(n, d) \leq \psi_{k}^{(i)}(n-1, d)+A n^{k-2} \log n
$$

where $A$ is a constant depending on $k, i, d$, and $\delta$. Since $k \geq 2$, this recurrence solves to $O\left(n^{k-1} \log n\right)$, yielding $\tau_{k}^{(i)}(n, d)=O\left(n^{d-1} \log n\right)$, with a constant of proportionality depending on $i, k, d, \delta$, as claimed.

To complete the argument, we need to extend this bound to the case $k=1$. For this we recall that $\tau_{k}^{(i)}(\sigma ; H)$ is the number of popular $(k, i)$-borders, i.e., the total number of $k$-faces of the popular $i$-faces in $\mathscr{Z}_{g}(H)$. Since we view our arrangement as lying in projective $d$-space, each popular $i$-face is a simple $i$-polytope, so the number of its faces of all dimensions is at most a constant multiple (depending on $i$ ) of the number of its $\lceil i / 2\rceil$-faces (see, for example, Problem 6.2 in [7], or [2]). Hence $\tau_{1}^{(i)}(\sigma ; H)=O\left(\tau_{\lceil i / 27}^{(i)}(\sigma ; H)\right)$, but since $i>2$ we have $\lceil i / 2\rceil>1$, which implies that $\tau_{1}^{(i)}(n, d)$ is also $O\left(n^{d-1} \log n\right)$. This completes the proof of the Extended Zone Theorem for surfaces of dimension $d-1$.

For the more general case of the zone of a $p$-dimensional algebraic or convex surface, for $0 \leq p<d$, let $\tau_{k}^{(i)}(n, d, p)$ be the maximum of $\tau_{k}^{(i)}(\sigma ; H)$ over all choices of $H$ and of a surface $\sigma$ of dimension $p$ which is either convex or algebraic of degree at most some small fixed $\delta$. The functions $\tau$ obey (2), but (1) is replaced by

$$
\tau_{k}^{(k)}(n, d, p)=O\left(n^{p}\right) \quad \text { for all } \quad 0 \leq k \leq d
$$

We again introduce $\psi_{k}^{(i)}(n, d, p)$ such that

$$
\tau_{k}^{(i)}(n, d, p)=\left(\begin{array}{c}
n \\
d-k
\end{array}\right) \psi_{k}^{(i)}(n, d, p),
$$

and obtain a relation identical to (3). We proceed by induction on $i$. Assume first $i \leq d-p$. In this case the number of popular $i$-faces is $\tau_{i}^{(i)}(n, d, p)=O\left(n^{p}\right)$. The maximum complexity of an $i$-polyhedron bounded by at most $n$ facets is $O\left(n^{\lfloor i / 2\rfloor}\right)=O\left(n^{\lfloor(d-p) / 2\rfloor}\right)$. Therefore, for every $k \leq i \leq d-p, \tau_{k}^{(i)}(n, d, p)=O\left(n^{\lfloor(d+p) / 2\rfloor}\right)$.

Assume now that $d-p$ is even. For $i>d-p$ we solve the recurrence (2) assuming $k>\Gamma(d-p) / 2\rceil$. Assume that the bound holds inductively, so $\psi_{k-1}^{(i-1)}(n, d, p)=O\left(n^{k-1-\Gamma(d-p) / 2 T}\right)$. Inserting this bound in (3), we obtain $\psi_{k}^{(i)}(n, d$, $p)=O\left(n^{k-\Gamma(d-p) / 2\rceil}\right)$ which gives the desired bound $\tau_{k}^{(i)}(n, d, p)=O(\lfloor(d+m) / 2\rfloor)$. For $k \leq\lceil(d-p) / 2\rceil$ we have $k \leq\lceil(d-p) / 2\rceil<\lceil i / 2\rceil$. As noted above, the number of faces of any dimension bounding a simple $i$-polytope is, up to a multiplicative constant, dominated by the number of its $\lceil i / 2\rceil$-faces, and since we are in projective 
space, popular $i$-borders are simple $i$-polytopes, so for all $k, \tau_{k}^{(i)}(n, d, p)=$ $O\left(\tau_{i j / 2}^{(i)}(n, d, p)\right)=O\left(n^{(d+p) / 2}-\right)$.

For $d-p$ odd, we first handle the case when $i=d-p+1, k=\lceil(d-p) / 2\rceil=$ $\left\lceil i / 27\right.$. By induction hypothesis, we have $\psi_{k-1}^{(i-1)}(n, d, p)=O\left(n^{k-1-\lceil(d-p) / 27}\right)=O\left(n^{-1}\right)$, so (3) solves to $\psi_{k}^{(i)}(n, d, p)=O(\log n)$, or $\tau_{k}^{(i)}(n, d, p)=O\left(n^{\lfloor(d+p) \backslash 2\rfloor} \log n\right)$. By the same reasoning that we used above, this implies that, for any $0 \leq k<i$, we have $\tau_{k}^{(i)}(n, d, p)=O\left(n^{\lfloor(d+p) / 2\rfloor} \log n\right)$. (Actually, for $k>\lceil i / 2\rceil$, (3) solves to $\psi_{k}^{(i)}(n, d, p)=O\left(n^{k-[(d-p) / 2\rceil}\right)$, so that $\tau_{k}^{(i)}(n, d, p)=O\left(n^{\lfloor(d+p), 2\rfloor}\right.$.) For $i \geq d-p+2$ we solve the recurrence for $k>\lceil(d-p) / 2\rceil$ as in the case of $d-p$ even, to obtain $\tau_{k}^{(i)}(n, d, p)=O\left(n^{\lfloor(d+p) / 2\rfloor} \log n\right)$ (where, again, if $k$ is sufficiently large, that is,

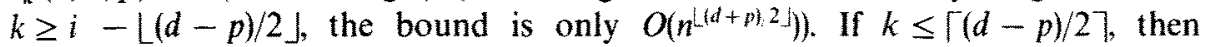
$k \leq\lceil(i-2) / 2\rceil<\lceil i / 2\rceil$ and again the Dehn-Sommerville relations extend the bound for all $0 \leq k \leq i$. This completes the proof of the Extended Zone Theorem.

\section{Discussion}

The following immediate application of the Extended Zone Theorem is obtained from an observation of Pellegrini [13], [14], as also discussed in [2]:

Theorem 3.1. Given $n$ triangles in three-dimensional space and any $\varepsilon>0$, we can preprocess them in randomized expected time $O\left(n^{4+\varepsilon}\right)$ into a data structure of size $O\left(n^{4+\varepsilon}\right)$, so that, for a query ray $\rho$, we can compute the first triangle met by $\rho$ in time $O(\log n)$.

This result improves the preprocessing and space complexity of the best previous solution, given in [2], by a factor of roughly $n^{1 / 2}$. A related application of the Extended Zone Theorem is also given by de Berg et al. [6].

Agarwal and Matoušek [1] have applied our result to the same problem, using a different technique, to obtain

Theorem 3.2. Given $n$ triangles in three-dimensional space and any $\varepsilon>0$, we can preprocess them in randomized expected time $O\left(n^{1+\varepsilon}\right)$ into a data structure of size $O\left(n^{1+\varepsilon}\right)$, so that, for a query ray $\rho$, the first triangle met by $\rho$ can be computed in time $O\left(n^{3 / 4+\varepsilon}\right)$.

These applications use only the Extended Zone Theorem for $p=d-1$ (the surface in question is the so-called Plücker surface, which is a four-dimensional quadric in $\mathbb{R}^{5}$; see, for example, [13]). It would be interesting to find applications of the theorem for $p<d-1$. In terms of further extending the Zone Theorem, we plan to investigate the class of surfaces for which the complexity of the zone in an arrangement of $n$ hyperplanes in $\mathbb{R}^{d}$ is close to $O\left(n^{d-1}\right)$. One immediate observation is that any surface whose intersection with an arbitrary $k$-flat in $\mathbb{R}^{d}$, $0<k \leq d$, has a bounded number of components falls into this category. Another intriguing and largely unexplored area is that of replacing hyperplane arrange- 
ments by arrangements of more general algebraic surfaces or some other classes of objects-one such situation is discussed in [4]. Finally, it would be interesting to settle the problem of whether the complexity of the zone of an algebraic surface in a hyperplane arrangement in $\mathbb{R}^{d}$ can be larger than $O\left(n^{d-1}\right)$. This problem is open even in the plane.

\section{References}

1. P. K. Agarwal and J. Matoušek, On range searching with semialgebraic sets, Proc. 17th Symp. on Mathematical Foundations of Computer Science, 1992, pp. 1-13. Lecture Notes in Computer Science, Vol. 629, Springer-Verlag, Berlin.

2. B. Aronov, J. Matoušek, and M. Sharir, On the sum of squares of cell complexities in hyperplane arrangements, Proc. 7th Symp. on Computational Geometry, 1991, pp. 307-313.

3. B. Aronov and $M$. Sharir, On the zone of a surface in a hyperplane arrangement, Proc. 2nd Workshop on Algorithms and Data Structures, Ottawa, 1991, pp. 13-19.

4. B. Aronov and M. Sharir, Castles in the air revisited, Proc. 8th Symp. on Computational Geometry, 1992, pp. 146-156.

5. M. Bern, D. Eppstein, P. Plassman, and F. Yao, Horizon theorems for lines and polygons. In Discrete and Computational Geometry: Papers from the DIMACS Special Year, J. Goodman, R. Pollack, and W. Steiger, eds., pp. 45-66. DIMACS Series in Discrete Mathematics and Theoretical Computer Science, Vol. 6, American Mathematical Society, Providence RI, 1991.

6. M. de Berg, D. Halperin, M. Overmars, J. Snoeyink, and M. van Kreveld, Efficient ray shooting and hidden surface removal, Proc. 7th Symp. on Computational Geometry, 1991, pp. 21-30.

7. H. Edelsbrunner, Algorithms in Combinatorial Geometry, Springer-Verlag, Heidelberg, 1987.

8. H. Edelsbrunner, L. Guibas, J. Pach, R. Pollack, R. Seidel, and M. Sharir, Arrangements of curves in the plane: Topology, combinatorics, and algorithms, Proc. 15th Internat. Colloq. on Automata, Languages and Programming, 1988, pp. 214-229.

9. H. Edelsbrunner, R. Seidel, and M. Sharir, On the zone theorem for hyperplane arrangements, SIAM J. Computing, to appear.

10. B. Grünbaum, Convex Polytopes, Wiley, New York, 1967.

11. M. E. Houle and T. Tokuyama, On zones of flats in hyperplane arrangements, Technical Report 92-3, Department of Information Science, Faculty of Science, University of Tokyo, April 1992.

12. J. Milnor, On the Betti numbers of real varieties, Proc. Amer. Math. Soc. 15 (1964), 275-280.

13. M. Pellegrini, Combinatorial and algorithmic analysis of stabbing and visibility problems in 3-dimensional space, Ph.D. Thesis, Courant Institute, New York University, February 1991.

14. M. Pellegrini, Ray-shooting and isotopy classes of lines in 3-dimensional space, Proc. 2nd Workshop on Algorithms and Data Structures, Ottawa, 1991, pp. $20-31$.

15. M. Pellegrini, On the zone of a codimension $p$ surface in a hyperplane arrangement, Proc. $3 r d$ Canadian Conference on Computational Geometry, Vancouver, 1991, pp. 233-238.

Received June 19, 1991, and in revised form March 25, 1992. 